\author{
Request for Comments on Request for Comments \\ Instructions to RFC Authors
}

Status of this Memo

This RFC specifies a standard for the Internet community. Authors of RFCs are expected to adopt and implement this standard. Distribution of this memo is unlimited.

1. Introduction

RFCs are distributed online by being stored as public access files, and a short message is sent to the distribution list indicating the availability of the memo.

The online files are copied by the interested people and printed or displayed at their site on their equipment. (An RFC may also be returned via email in response to an email query.) This means that the format of the online files must meet the constraints of a wide variety of printing and display equipment.

\title{
2. Format Rules
}

To meet the distribution constraints the following rules are established for the two allowed formats for RFCs: ASCII and Post Script.

The RFC Editor attempts to ensure a consistent RFC style. To do this the RFC Editor may choose reformat the RFC submitted. It is much easier to do this if the submission matches the style of the most recent RFCs. Please do look at some recent RFCs and prepare yours in the same style.

You must submit an editable online document to the RFC Editor. The RFC Editor may require minor changes in format or style and will insert the actual RFC number.

2a. ASCII Format Rules:

The character codes are ASCII.

Each page must be limited to 58 lines followed by a form feed on a 
line by itself.

Each line must be limited to 72 characters followed by carriage return and line feed.

No overstriking (or underlining) is allowed.

These "height" and "width" constraints include any headers, footers, page numbers, or left side indenting.

Do not fill the text with extra spaces to provide a straight right margin.

Do not do hyphenation of words at the right margin.

Do not use footnotes. If such notes are necessary, put them at the end of a section, or at the end of the document.

Use single spaced text within a paragraph, and one blank line between paragraphs.

RFCs in ASCII Format may be submitted to the RFC Editor in email messages (or as online files) in either the finished publication format or in NROFF. If you plan to submit a document in NROFF, please consult the RFC Editor first.

2b. Postscript Format Rules

Standard page size is $81 / 2$ by 11 inches.

Margin of 1 inch on all sides (top, bottom, left, and right). Main text should have a point size of no less than 10 points with a line spacing of 12 points.

Footnotes and graph notations no smaller than 8 points with a line spacing of 9.6 points.

Three fonts are acceptable: Helvetica, Times Roman and Courier Plus their bold-face and italic versions. These are the three standard fonts on most Postscript printers.

Prepare diagrams and images based on lowest common denominator PostScript. Consider common Postscript printer functionality and memory requirements.

The following Post Script commands should not be used:

initgraphics, erasepage, copypage, grestoreall, initmatrix, 
initclip, banddevice, framedevice, nulldevice and renderbands.

These PostScript rules are likely to changed and expanded as experience is gained.

RFCs in PostScript Format may be submitted to the RFC Editor in email messages (or as online files). Since Postscript is not editable, an editable source version of the document must also be submitted. If you plan to submit a document in Postscript, please consult the RFC Editor first.

\section{Status statement}

Each RFC must include on its first page the "Status of this Memo" section which contains a paragraph describing the intention of the RFC. This section is meant to convey the status granted by the RFC Editor and the Internet Activities Board (IAB). There are several reasons for publishing a memo as an $\mathrm{RFC}$, for example, to make available some information for interested people, or to begin or continue a discussion of an interesting idea, or to make available the specification of a protocol.

The following sample paragraphs may be used to satisfy this requirement :

Proposed Protocol

This RFC suggests a proposed protocol for the Internet community, and requests discussion and suggestions for improvements.

Specification

This RFC specifies a standard for the Internet community. Hosts on the Internet are expected to adopt and implement this standard.

Discussion

The purpose of this RFC is to focus discussion on particular problems in the Internet and possible methods of solution. No proposed solutions this document are intended as standards for the Internet. Rather, it is hoped that a general consensus will emerge as to the appropriate solution to such problems, leading eventually to the adoption of standards. 


\section{Information}

This RFC is being distributed to members of the Internet community in order to solicit their reactions to the proposals contained in it. While the issues discussed may not be directly relevant to the research problems of the Internet, they may be interesting to a number of researchers and implementers.

Status

In response to the need for maintenance of current information about the status and progress of various projects in the Internet community, this RFC is issued for the benefit of community members. The information contained in this document is accurate as of the date of publication, but is subject to change. Subsequent RFCs will reflect such changes.

These paragraphs need not be followed word for word, but the general intent of the RFC must be made clear.

4. Distribution Statement

Each RFC is to also include a "distribution statement". In general, RFCs have unlimited distribution. There may be a few cases in which it is appropriate to restrict the distribution in some way.

Typically, the distribution statement will simply be the sentence "Distribution of this memo is unlimited." appended to the "Status of this Memo" section.

\section{Author's Address}

Each RFC must have at the very end a section giving the author's address, including the name and postal address, the telephone number, and the Internet email address.

6. Relation to other RFCs

Sometimes an RFC adds information on a topic discussed in a previous RFC or completely replaces an earlier RFC. There are two terms used for these cases respectively, UPDATES and OBSOLETES. A document that obsoletes an earlier document can stand on its own. A document that merely updates an earlier document cannot stand on its own; it is something that must be added to or inserted into the existing document, and has limited usefulness independently. The terms SUPERSEDES and REPLACES are no longer used. 
UPDATES

To be used as a reference from a new item that cannot be used alone (i.e., one that supplements a previous document), to refer to the previous document. The newer publication is a part that will supplement or be added on to the existing document; e.g., an addendum, or separate, extra information that is to be added to the original document.

OBSOLETES

To be used to refer to an earlier document that is replaced by this document. This document contains either revised information, or else all of the same information plus some new information, however extensive or brief that new information is; i.e., this document can be used alone, without reference to the older document.

For example:

On the Assigned Numbers RFCs, the term OBSOLETES should be used since the new document actually incorporates new information (however brief) into the text of existing information and is more up-to-date than the older document, and hence, replaces it and makes it OBSOLETE.

In lists of RFCs or the RFC-Index (but not on the RFCs themselves), the following may be used with early documents to point to later documents.

OBSOLETED-BY

To be used to refer to the newer document that replaces the older document.

UPDATED-BY

To be used to refer to the newer document that adds information to the existing, still useful, document. 
7. The RFC Editor

The RFC Editor is Jon Postel.

8. The RFC Announcement List

New RFCs are announced to the RFC distribution list maintained by the SRI Network Information Center (NIC). Contact the SRI-NIC to be added or deleted from this mailing list by sending an email message to RFC-REQUEST@NIC.DDN.MIL.

9. Obtaining RFCs

RFCs can be obtained via FTP from NIC.DDN.MIL, with the pathname RFC:RFCnnnn.TXT (where "nnnn" refers to the number of the RFC). Login with FTP, username ANONYMOUS and password GUEST.

The NIC also provides an automatic mail service for those sites which cannot use FTP. Address the request to SERVICEQNIC.DDN.MIL and in the subject field of the message indicate the RFC number, as in "Subject: RFC nnnn".

Requests for special distribution (for example, hardcopy) should be addressed to either the author of the RFC in question, or to NIC@NIC.DDN.MIL.

Unless specifically noted otherwise on the RFC itself, all RFCs are for unlimited distribution.

The RFCs may also be obtained from other information centers, including the CSNET Information Center (INFO@SH.CS.NET), the NSFNET Information Service (INFO@NIS.NSF.NET).

Author's Address

Jon Postel

USC Information Sciences Institute

4676 Admiralty Way

Marina del Rey, California 90292-6695

Phone: 213-822-1511

EMail: POSTELQISI.EDU 